\title{
Design für Alle: Attraktiv und hilfreich
}

Mathias Knigge

Produkte, Architektur und Dienstleistungen können durch altersbedingte Veränderungen schwer nutzbar sein. Der demografische Wandel erfordert eine neue Herangehensweise: Anstelle spezieller/defizit-orientierter Lösungen für wenige Konsumenten, die häufig als stigmatisierend empfunden werden, ist es sinnvoll, klare Mehrwerte für grosse Gruppen zu schaffen. Das Konzept «Design für Alle» bietet hier einen Ansatz, um attraktive Angebote zu entwickeln - unabhängig von Alter oder Behinderung.

\section{Le design pour tous : attrayant et utile}

Les produits, l'architecture et les prestations peuvent être difficiles à utiliser en raison des changements liés à l'âge. L'évolution démographique exige en effet une nouvelle approche : à la place de solutions spéciales/orientées sur les déficiences pour un petit nombre de consommateurs, qui sont fréquemment ressenties comme stigmatisantes, il est judicieux de créer des plus-values claires pour de grands groupes. Le concept « Design pour tous » propose en ce sens une démarche afin de développer des offres attractives indépendamment de l'âge ou du handicap.

DOI 10.1024/2297-5160/a000037

U nsere Gesellschaft verändert sich. Hersteller, Dienstleister, Handel und Tourismus stehen in Zukunft zunehmend älteren oder behinderten Kunden gegenüber und müssen auf deren spezifische Bedürfnisse eingehen.

\section{Inklusion als Chance für Unternehmen}

Attraktive Angebote sind auf einmal die, die nicht nur gut aussehen, sondern auch durch leichte Nutzbarkeit überzeugen, ohne als Seniorenprodukt oder Hilfsmittel wahrgenommen zu werden. Somit sind Inklusion und der

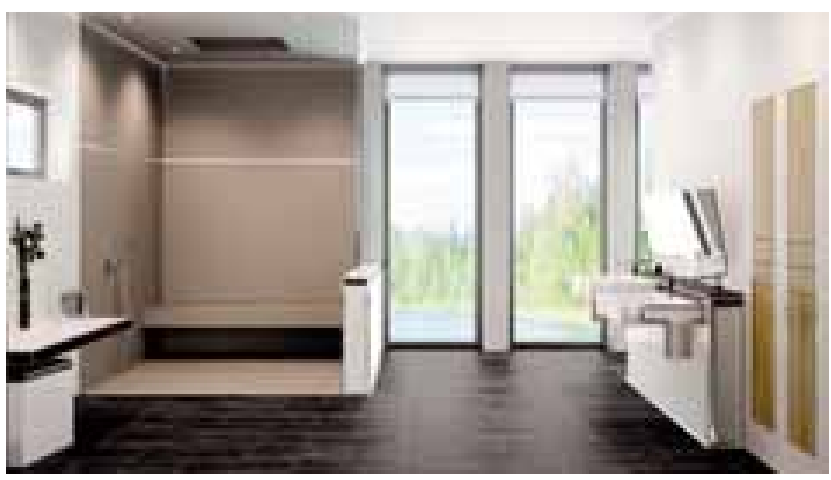

Bodengleiche Dusche

demografische Wandel, bei allen damit verbundenen Schwierigkeiten, für Unternehmen eine grosse Chance, sich mit ansprechenden Lösungen abseits von speziellen Seniorenprodukten zu positionieren.

\section{Defizitorientierte Lösungen haben bei der Mehrheit keine Chance}

Dies lässt sich gut am Beispiel barrierefreier Bäder veranschaulichen. Wenn nur funktionale Aspekte zur Vermeidung von Barrieren berücksichtigt werden, entstehen wenig attraktive Räume mit gross dimensionierten Klappgriffen und weit vorstehenden Toilettenbecken. Dies weckt Vorbehalte nicht nur bei älteren Konsumenten. Gut gestaltete Lösungen, wie bodengleiche Duschen, sind allein aufgrund der damit verbundenen Konnotation «Wellness/Hotel» für viele attraktiv. Sie ermöglichen aber die Zugänglichkeit mit Rollstuhl oder Rollator und sind auf diese Weise ein wichtiger Mehrwert für ältere oder behinderte Menschen (Knigge 2011a,b; 2013).

\section{Beispiele attraktive Lösungen im Design für Alle aus der Praxis}

Die Kunsthalle Emden entwickelte ein Demografiekonzept mit dem Ziel, Barrieren zu entfernen und attraktiven Komfort bei der Kunstbetrachtung zu schaffen. Ein ebener Zugang, leserliche Beschriftungen, aber auch erhöhte Sitzgelegenheiten gehörten dazu. grawwert unterstützte das Museum für eine langfristige Umsetzung dieser Anpassungen mit einem Planungshandbuch. 


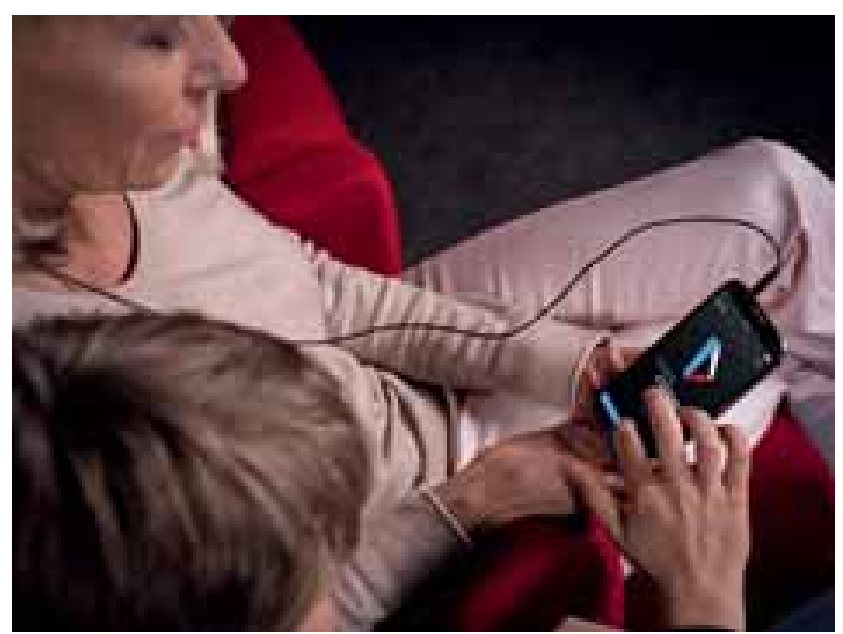

CinemaConnect

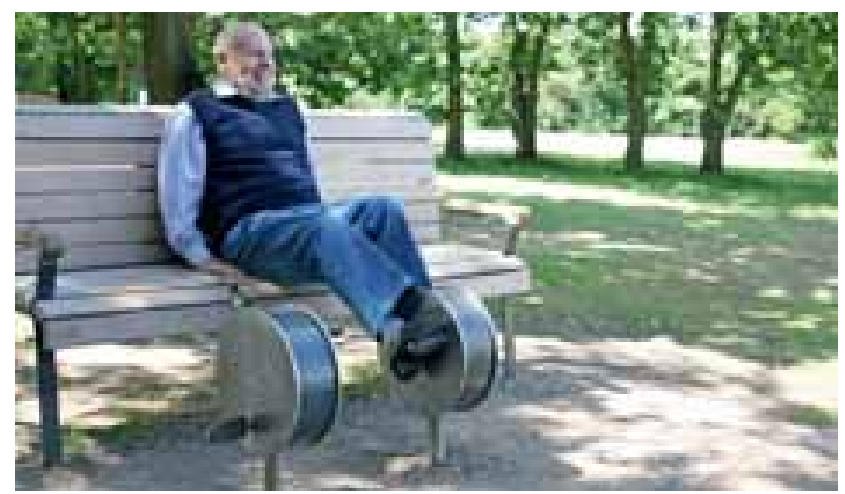

Radtrainer

Um kulturelle Veranstaltungen auch bei Hör- oder Seheinschränkungen zugänglich zu machen, wurden die Smartphone-Apps «MobileConnect» und «CinemaConnect» entwickelt. Die Apps bieten Hörunterstützung für schwerhörige Menschen im Theater und Kino. Bei Sehbehinderung ist eine Szenenbeschreibung (Audiodeskription) verfügbar. So können auch Untertitel und Sprachversionen (Originalsprache) im Kinosaal erlebt werden. Die Nutzung des Apps kann dabei individuell und unauffällig erfolgen, was für die Nutzer sowie die anderen Zuschauer besonders wichtig ist (Knigge, 2014). Ein attraktiver Mehrwert im Design für Alle.

Mit dem Bewegungsparcours Giro Vitale entstand eine attraktive Möglichkeit zur Gesundheitsförderung im öffentlichen Raum. Das Angebot überfordert nicht und lädt zu kleinen Übungen ein: Radtrainer, Balancebrett oder Nordic-Trainer bieten eine gute Möglichkeit, um in Eigenmotivation etwas für die Gesundheit zu tun und werden seit vielen Jahren erfolgreich in Parks und im Umfeld von Wohnanlagen eingesetzt. So wird für Erhalt der Gesundheit bis ins hohe Alter ein wichtiger Beitrag geleistet. Ein Mehrwert, der auch in einer Studie der Charité Berlin nachgewiesen werden konnte (Blümke, 2014). Aktuell untersucht grauwert, ob dieses Bewegungsangebot zur selbstständigen Nutzung im Umfeld von Klinik oder Reha-Zentrum für rehabilitierende Patientinnen und Patienten klassische Angebote, wie Ergotherapie und Physiotherapie, sinnvoll ergän- zen kann. Der informelle Ansatz ermöglicht attraktive Aussenanlagen mit einem gesundheitlichen Mehrwert zu verbinden - so kann Gesundheitsförderung im Design für Alle aussehen.

\section{Schritte, die attraktive Lösungen mit barrierefreier Nutzung verbinden}

Um Unternehmen für das Thema zu gewinnen, hat der Autor gemeinsam mit Partnern aus dem Kompetenznetzwerk «Design für Alle - Deutschland» (EDAD) eine Studie für das Bundeswirtschaftsministerium (BMWi) erstellt (Neumann, 2014). Sie zeigt, wie sinnvoll der Perspektivwechsel von einer Defizitbetrachtung zum Gewinn eines Mehrwerts ist. Hinzu kommt, dass dieser Perspektivwechsel angesichts des demografischen Wandels auch attraktive wirtschaftliche Potenziale birgt, die so zu einer win-win-Situation für Wirtschaft und Gesellschaft werden kann.

Aus der Praxiserfahrung des Autors ist für einen solchen Perspektivwechsel eine umfassende Beratung nötig. Diese reicht von konkreten Situationsanalysen und Nutzertests, über Konzepte bis hin zur Entwicklungsbegleitung. Grundlage für entsprechende Veränderungen sind sehr häufig Schulungen und Sensibilisierungsmassnahmen. Dafür wird zum Beispiel mit dem grauwert-Simulationsanzug ein Gespür für diverse Nutzerbedürfnisse vermittelt, was gerade für jüngere Entwickler wichtig ist, um das Potenzial eines Design für Alle ganz direkt und selbst zu erleben (Knigge 2011).

\section{Literatur}

Blümke, C., Leppert, U. \& Ramirez J.P. (2014) SNAP - Studie zum Nutzungsverhalten des Bewegungs-Parcours Giro Vitale durch Senioren. Zeitschrift für Gerontologie und Geriatrie, 47(1), 150.

Knigge, M. (2013) Produktentwicklung für Alt und Jung. In: Schirmacher: Chancen des demographischen Wandels für Konsum und Finanzen. Frankfurt: Frankfurter Allgemeine Buch.

Knigge M. \& Erkau J. (2014) Cinema and Theatre: Accessible Entertainment, with Benefits for All. In: UD 2014, Proceedings of the international conference on Universal Design 2014, Lund. Amsterdam: IOS Press, 421-422.

Neumann P., Knigge M., et.al (2014) Entwicklung Handlungsleitender Kriterien für KMU zur Berücksichtigung des Konzepts Design für Alle in der Unternehmenspraxis. Studie im Auftrag des Bundesministeriums für Wirtschaft und Energie (vorm. Bundesministerium für Wirtschaft und Technologie), Hamburg/Münster: BMWi.

Weiterführende Literatur kann beim Autor bezogen werden.

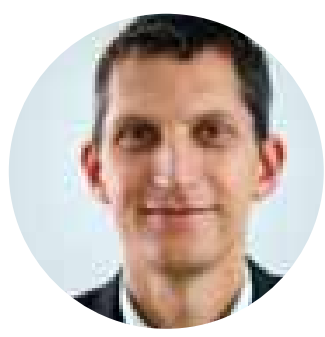

Mathias Knigge hat 2004 «grauwert Büro für Inklusion und demografiefeste Lösungen» in Hamburg gegründet (www.grauwert.info). Er ist Vorstandsmitglied von EDAD (Design für Alle - Deutschland e.V.).

m.knigge@grauwert.info 